\title{
Investigating student understanding of perturbation theory and inner products of functions
}

\author{
Gina Passante, Paul J. Emigh, Tong Wan, and Peter S. Shaffer \\ Department of Physics, University of Washington, Seattle, Washington 98195
}

\begin{abstract}
We have investigated the extent to which students can qualitatively determine the effect of perturbations to a Hamiltonian on the energies of the eigenfunctions. The results indicate that after lecture instruction many students cannot determine some important features of the first-order correction. We examine the possibility that this failure may stem from a lack of understanding of the inner product. Since perturbations are often represented graphically, the focus has been on student ability to determine the inner product of functions represented graphically. In the process, we have found that some students are unable to find inner products even in contexts outside of quantum mechanics.
\end{abstract}

PACS numbers: 01.40.G-, 03.65.Ta, 03.65.-w

\section{INTRODUCTION}

Many undergraduate quantum mechanics courses teach the basics of quantum mechanics using ideal systems. Approximation methods, such as perturbation theory, allow the use of solutions to the Schrödinger equation for simple potentials in solving more realistic experimental systems. We present preliminary results from an investigation into student conceptual understanding of the first-order correction to the energy and of the inner product.

In quantum mechanics, inner products are used to calculate the probabilities of different measurement outcomes, and in perturbation theory, the corrections to the energy. Students must be able to find the inner products of both vectors and functions. There has been some research on student understanding of addition, subtraction, and scalar multiplication of vectors [1-4], and of the dot product [5-7], but very little, if any work has looked at student ability to perform inner products on functions in the context of physics.

This paper begins in the context of perturbation theory where we investigate student ability to determine the sign and relative magnitude of energy corrections for small perturbations to the infinite square well. The potentials are represented graphically as the questions were designed to have students focus on qualitative features of the inner products, such as whether they are positive, negative, or zero. We then discuss student performance on questions that require taking inner products of functions in different contexts.

\section{CONTEXT FOR INVESTIGATION}

This investigation has taken place primarily in a two-quarter sequence of junior-level quantum mechanics (QM) courses at the University of Washington (UW). The first quarter is required for all physics majors, and the second is taken by approximately half of all physics majors. The sequence covers the entire Introduction to Quantum Mechanics textbook by D. J. Griffiths. Both courses have 2.5 hours of lecture and a 50-minute small-group section each week. The small-group sections are run by graduate teaching assistants and use $T u$ - torials in Physics: Quantum Mechanics [8]. These tutorial address many of the findings from investigations by our group and others into student understanding of quantum mechanics, but do not yet address the issues raised in this paper.

The data analyzed in this work are all from weekly online surveys that focus on material related to the lectures and tutorials throughout the course. They are in multiple-choice format followed by requests for explanations of reasoning. The questions are generally asked after relevant lecture instruction, but before tutorial instruction. Responses are not graded for correctness, but students are given participation credit for answering the questions. In our experience students take the pretests seriously, but do not always give detailed explanations. One of the questions was administered in a junior-level electricity and magnetism (EM) class. The student populations in the QM and EM course are very similar and many students take them concurrently.

Our research perspective assumes that student responses to written questions can provide insight into their level of understanding. We believe that by removing some of the more complex components of the questions, thereby focusing the questions on fewer concepts, we can identify which concepts are most difficult for students and gain insight into the underlying problems.

\section{STUDENT UNDERSTANDING OF PERTURBATION THEORY}

At UW, perturbation theory is taught in the second quarter of the two-course sequence on quantum mechanics. Figure 1 shows several questions asked on an online survey in this course. The questions provide students with a graphical representation of two perturbations to the infinite square well and ask whether the first-order correction to the energy of the lowest two (or three) energy eigenstates is positive, negative, or zero for each perturbation. Students are also asked whether the magnitude of one correction is greater than, less than, or equal to the magnitude of another correction. The questions provide students with the equation for the first-order correction 


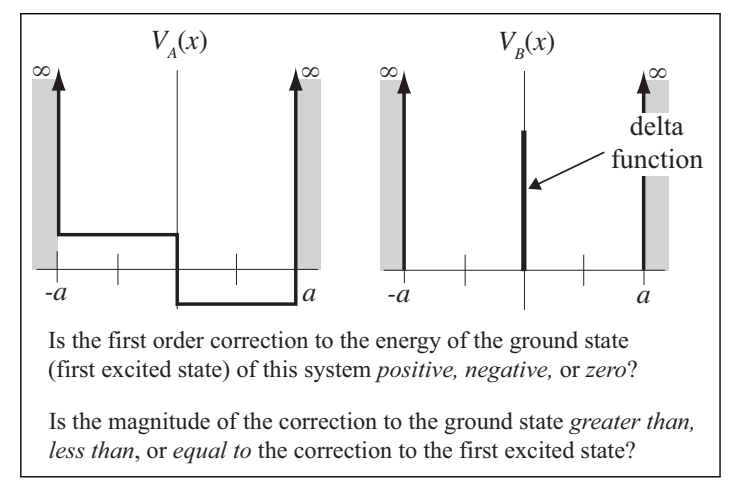

FIG. 1. Questions for two perturbed infinite square wells of width $2 a$ centered at $x=0$. Students were asked to answer the questions for each well independently. For potential $V_{B}(x)$ students were also asked the same questions for the second excited state.

to the energy:

$$
E_{n}^{(1)}=\left\langle\psi_{n}\left|H^{\prime}\right| \psi_{n}\right\rangle
$$

where $H^{\prime}$ is the perturbation to the Hamiltonian, and $\psi_{n}$ is an energy eigenfunction of the unperturbed Hamiltonian with energy eigenvalue $E_{n}^{(0)}$.

To solve for the correction to the energy, it is helpful for students to recognize that Eq. 1 can be re-written as an integral in position space:

$$
E_{n}^{(1)}=\int \psi_{n}^{*}(x) H^{\prime}(x) \psi_{n}(x) d x .
$$

It is then possible for students to integrate the product of the functions qualitatively to determine if the first-order correction to the energy is positive, negative, or zero. For example, $V_{A}(x)$ is anti-symmetric about $x=0$ while the probability density for each energy eigenfunction is positive and symmetric about the same point. Thus the first-order correction to each energy eigenvalue is zero. For $V_{B}(x)$, however, only the value of the probability density at $x=0$ has an effect on the first-order correction to the energy. The first-order correction to the first excited-state energy is thus zero, while the energy corrections of the ground state and second-excited state are equal and positive. This is one example of the type of qualitative explanation we would like students to provide.

The results are summarized in Table 1. About half of the students determined the correct sign of each correction, except for $E_{2}^{(1)}$ for $V_{A}(x)$, where approximately $35 \%$ answered correctly and $40 \%$ incorrectly stated that the correction would be positive. Some of the latter students did not square the wave function, and therefore obtained a positive value for the integral over both the right and left half of the well.

Some students gave incorrect reasoning to support the correct answer. For this reason, we use student performance on pairs of questions as a proxy for student understanding. The last column of Table 1 gives the percentage of the students who answered each question correctly for both potentials. The bottom row refers to students who correctly answered the sign of
TABLE I. Percentage of the students correctly answering the perturbation theory questions in Fig. 1. The total number of students who answered each question correctly is in parentheses. $(N=95)$

\begin{tabular}{c|c|c|c}
\hline \hline & $\boldsymbol{V}_{\boldsymbol{A}}(\boldsymbol{x})$ & $\boldsymbol{V}_{\boldsymbol{B}}(\boldsymbol{x})$ & Both \\
\hline$E_{1}^{(1)}$ & $50 \%(49)$ & $60 \%(58)$ & $35 \%(33)$ \\
$E_{2}^{(1)}$ & $35 \%(34)$ & $55 \%(53)$ & $25 \%(22)$ \\
$E_{3}^{(1)}$ & N/A & $45 \%(44)$ & N/A \\
\hline$E_{1}^{(1)}$ vs. $E_{2}^{(1)}$ & $50 \%(49)$ & $60 \%(58)$ & $35 \%(33)$ \\
$E_{1}^{(1)}$ vs. $E_{3}^{(1)}$ & N/A & $45 \%(43)$ & N/A \\
\hline All energies correct & $30 \%(27)$ & $20 \%(21)$ & $10 \%(9)$ \\
\hline \hline
\end{tabular}

all two (or three) energy corrections. As might be expected, these percentages are much lower, often less than half of that for students correct on a single question.

One reason for the poor performance might be that students are unable to go from Eq. 11 to Eq. 2, that is, they cannot successfully convert Dirac notation into position space. Another possibility is that students are unable to interpret the integral equation when only given graphical functions. The next section probes the latter possibility further.

\section{STUDENT UNDERSTANDING OF INNER PRODUCTS}

In order to further investigate the difficulties that students have with perturbation theory, we asked three sets of questions that require students to find the inner products of functions represented graphically (see Fig. 2). Contexts A and B were administered in the QM course and context $\mathrm{C}$ was administered in a junior-level EM course (as described in Section III).

\section{A. Context A: Probabilities in QM}

In context $\mathrm{A}$, students are given the graph of an energy eigenfunction with energy eigenvalue $E$. They are also given the graphs of three other wave functions, each corresponding to a different particle. Students are asked which wave function is most (and least) likely to be measured to have energy $E$. In order to answer this question correctly, students should recall that the probability of measuring a given energy is the modulus square of the inner product of the wave function with the corresponding energy eigenfunction. They should find that the probability for $\varphi_{a}$ and $\varphi_{c}$ are both zero.

TABLE II. Percentage of the students who selected each answer in context A. The correct answer is bolded. $(N=124)$

\begin{tabular}{c|c|c|c|c}
\hline \hline & A & B & C & More than one \\
\hline Most likely & $55 \%(66)$ & $\mathbf{2 0} \%(\mathbf{2 7})$ & $5 \%(9)$ & $5 \%(6)$ \\
Least likely & $15 \%(18)$ & $25 \%(31)$ & $30 \%(39)$ & $\mathbf{2 0 \% ( 2 4 )}$ \\
\hline \hline
\end{tabular}

Many students did not explicitly calculate an inner product, 


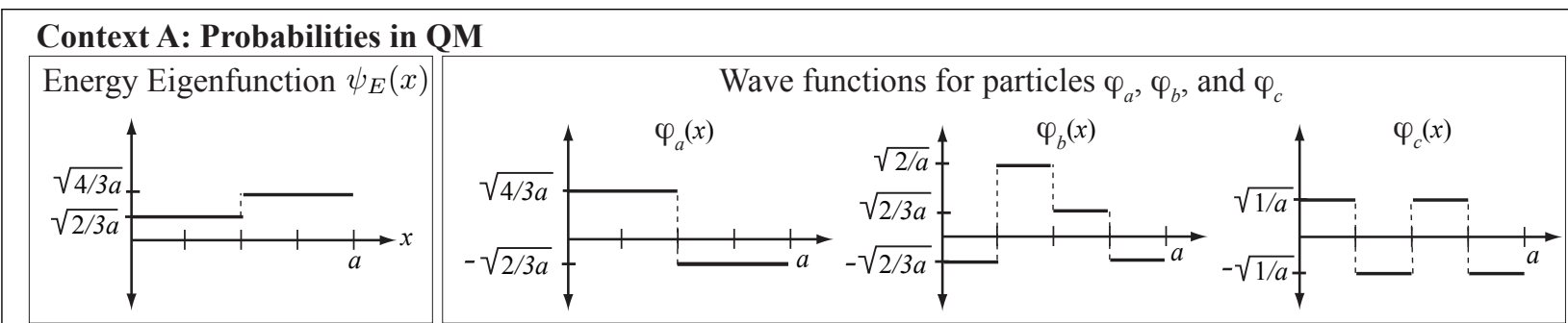

A1. Which particle is most likely to have measured energy E? A2. Which particle is least likely to have measured energy E?

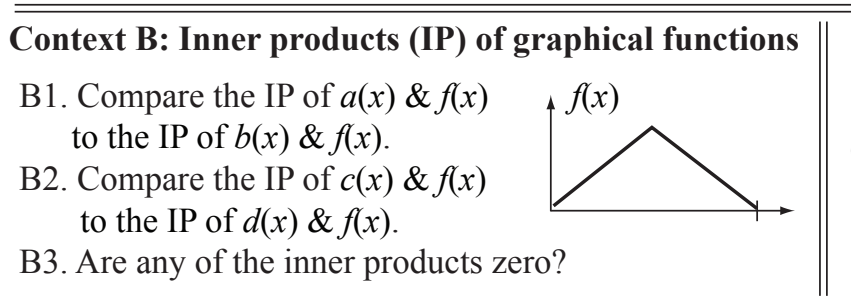

$a(x)$

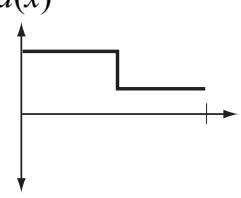

$b(x)$

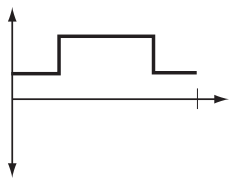

Context C: Integrals of graphical functions

C1. Determine whether each integral is positive, negative, or zero.
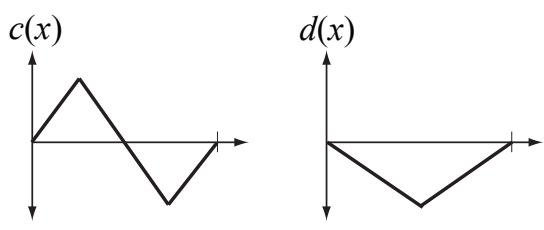

$J_{c d}=\int c(x) d(x) d x$

$J_{d e}=\int d(x) e(x) d x$

$J_{c e}=\int c(x) e(x) d x$ $e(x)$

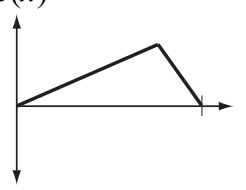

FIG. 2. Abbreviated versions of inner product questions in three different contexts. Context A provides students with an energy eigenfunction and asks which of three wave functions is most (least) likely to be measured to have the associated energy. In context B students are asked whether one inner product was greater than, less than, or equal to another inner product of functions that are represented graphically. Finally, in context $\mathrm{C}$ students are asked to determine whether an integral is positive, negative, or zero. All versions were multiple choice and also asked students to explain their reasoning in a text box below the answer choices.

but rather looked for similarities in the shapes of the functions. While the shape of a function can be useful information for determining qualitative features of the inner product, many students were focusing on some less important surface features of the graphs. For example, over half of the students indicated that particle A is most likely to be measured to have energy $E$. Many of these students explained that this was because the graphs are similar in that they both have two segments (with one discontinuity), or that both functions have levels at $\sqrt{4 / 3 a}$ and $\pm \sqrt{2 / 3 a}$. About $40 \%$ of the students used this type of reasoning for question $\mathrm{A} 1$ and $35 \%$ for question $\mathrm{A} 2$.

Correctly answering this question requires multiple steps: recognizing the need for an inner product, knowing how to perform an inner product for functions, and evaluating the integral of a product of functions represented graphically. From these questions it is not clear the extent to which each step is causing difficulty. The next two sets of questions probe whether or not student performance improves as the questions become more targeted.

\section{B. Context B: Inner Products of Graphical Functions}

In context $\mathrm{B}$, we directly probe student ability to calculate the inner product of functions that are represented graphically. Two of the questions explicitly ask students whether one inner product is greater than, less than, or equal to the second inner product. The third question asks if any function has an inner product of zero with the test function. The question also provides students with the definition of the inner product of functions in words ("the inner product of two functions is the integral of the product of those functions"), but the equation was not provided. Table III shows the results from these questions.

TABLE III. Percentage of the students who provided the correct answer and correct reasoning to the questions in context $\mathrm{B}$. $(N=77)$

\begin{tabular}{c|c|c|c}
\hline \hline & B1 & B2 & B3 \\
\hline Correct answer & $60 \%(46)$ & $65 \%(51)$ & $50 \%(39)$ \\
and reasoning & $35 \%(27)$ & $45 \%(33)$ & $45 \%(36)$ \\
\hline \hline
\end{tabular}

In response to these questions, many students arrived at the correct answer but did not use correct reasoning. Relatively few students stated that they were comparing the shape of the wave functions in order to compare the inner products (as was common in context A). This may be a result of the particular set of functions that we presented, or it could be due to the fact that we asked students to find the inner product (instead of the probability of an energy measurement). Some students compared the "shared area" of the two graphs, indicating that two graphs that have more overlapping area will have a larger 
inner product. This procedure results in a correct answer for several questions, but incorrectly predicts that the inner product of $f(x)$ and $d(x)$ is zero. This was the most common incorrect answer: approximately $10 \%$ of the students reasoned that the inner product is zero because the functions are "equal and opposite", while a further $10 \%$ reasoned that the area of $d(x)$ cancels out the area of $f(x)$.

\section{Context C: Integrals of Graphical Functions}

In the final context, students were asked to evaluate the integrals of products of functions (provided as equations). The term "inner product" was not used. (For research on similar questions, see Ref [9] and references therein.) The question was asked on a junior-level EM online survey similar to those used in QM. The results are shown in Table IV] Student performance is similar to that on the questions in context B: almost half of the students were unable to give the correct answer. We found that most students did not provide detailed explanations for why they selected a particular answer.

TABLE IV. Percentage of the students who chose each answer option for the integrals in context $\mathrm{C}$. The correct answer for each integral is bolded. $(N=79)$

\begin{tabular}{c|c|c|c}
\hline \hline & Positive & Negative & Zero \\
\hline$J_{c d}$ & $5 \%(6)$ & $20 \%(18)$ & $\mathbf{6 5 \%}(\mathbf{5 2})$ \\
$J_{c e}$ & $25 \%(20)$ & $\mathbf{6 0} \%(\mathbf{4 7})$ & $10 \%(8)$ \\
$J_{d e}$ & $25 \%(19)$ & $\mathbf{5 0 \%}(\mathbf{4 1})$ & $15 \%(12)$ \\
\hline \hline
\end{tabular}

\section{DISCUSSION}

Successful understanding of perturbation theory requires working knowledge of inner products in quantum mechanics. One complication is that the equations for perturbation theory are often expressed in Dirac notation and must be converted to integrals for position-space wave functions. We have found that many students cannot approximate the correction to the energy qualitatively for a perturbation represented graphically. However, it is unclear whether the problems students have with these questions are due to the context of perturbation theory, or to an inability to take the inner product of functions.

As a first step to answering this question we looked at the responses to a variety of questions that require some subset of the skills needed to answer the perturbation theory questions. We found that questions asking students to solve for the probability of an energy measurement (which requires them to evaluate an inner product) are very difficult when the wave functions are represented graphically. These questions were the most difficult for students.

Student performance was better on questions that were not in the context of quantum mechanics. Approximately 50-65\% of the students answered correctly when they were asked to calculate the inner product qualitatively (context B) or were asked to calculate the integral of products of functions represented graphically (context C).

We found that on a variety of questions students had a tendency to compare superficial features of the wave functions (such as the number of levels, nodes, or discontinuities), the sign, or the area, instead of performing the inner product. For questions relating to energy measurements (context A) this could partially be due to the experience students have in visually inspecting wave functions for simple potentials (such as the infinite square well and the harmonic oscillator). However, this would not account for student performance on the questions in the more mathematical contexts (B and C).

These results indicate that questions involving inner products of functions are difficult for students. However, further research is needed to understand the underlying reasons and why some do not correctly apply their knowledge of inner products to quantum mechanics problems.

\section{ACKNOWLEDGMENTS}

We would like to thank UW faculty members John Rehr, Boris Blinov, and Leslie Rosenberg for welcoming this research in their courses. This research has been supported in part by the NSF under Grant No. DUE-1022449.
[1] R. D. Knight, The Physics Teacher 33, 74 (1995).

[2] P. Barniol and G. Zavala, in 2011 Phys. Educ. Res. Conf., edited by N. S. Rebello, C. Singh, and P. V. Engelhardt (Omaha, NE, 2012) p. 115.

[3] J. M. Hawkins, J. R. Thompson, M. C. Wittmann, E. C. Sayre, and B. W. Frank, in 2010 Phys. Educ. Res. Conf., edited by C. Singh, N. S. Rebello, and M. Sabella (Portland, OR, 2011) p. 165.

[4] N.-L. Nguyen and D. E. Meltzer, Am. J. Phys. 71, 630 (2003).

[5] J. Van Deventer, Comparing Student Performance on Isomorphic Math and Physics Vector Representations, Master's thesis, University of Maine (2008).
[6] G. Zavala and P. Barniol, in 2012 Phys. Educ. Res. Conf., edited by P. V. Englehardt, A. D. Churukian, and N. S. Rebello (Philadelphia, PA, 2013) p. 438.

[7] P. Barniol and G. Zavala, Phys. Rev. ST Phys. Educ. Res. 10, 020115 (2014).

[8] L. C. McDermott, P. S. Shaffer, P. R. L. Heron, and the UW Physics Education Group, Tutorials in Physics: Quantum Mechanics, preliminary ed. (2013).

[9] R. R. Bajracharya, Student Understanding of Definite Integrals with Relevance to Physics Using Graphical Representations, Master's thesis, University of Maine (2012). 